\title{
Cortical and cervical stimulation after hemispheric infarction
}

\author{
A BERARDElli,* M INGHilleri, ${ }^{*}$ M MANFREDI,* A ZAMPONI, $\dagger$ V CECCONI, $\dagger$ \\ G DOLCE $\dagger$
}

\author{
From the V Clinica Neurologica, Centro Disordini del Movimento, Dipartimento di Scienze Neurologiche, \\ Universita' di Roma "La Sapienza"," and Istituto San Giovanni Battista, $\dagger$ Roma, Italy
}

SUMMARY Cortical and cervical stimulation has been performed in 20 patients with hemiparesis or hemiplegia due to hemispheric infarction and in 20 control subjects. The motor action potentials (MAPs) were recorded from biceps and thenar muscles. MAPs evoked by stimulation of the undamaged hemisphere were normal in 18 out of 20 patients and in two there was a slight increase of central conduction time (CCT). The stimulation of the motor cortex of the damaged hemisphere did not evoke any response in 15 patients; in two the MAPs were absent in one muscle and in the remaining three were delayed in one or both muscles. Cervical MAPs were normal in 18 patients and delayed in the thenar muscle in two patients.

In the intact man various attempts have been made to stimulate the brain through the scalp, but owing to the considerable electrical resistance and high cortical threshold they have met with little success. Gualtierotti and Paterson ${ }^{1}$ tried repetitive stimulation of the scalp, which was abandoned because of local pain. Only in 1980, Mertón and Morton ${ }^{2}$ succeeded in activating contralateral muscles by applying to the scalp a single, short-lasting, high voltage electrical stimulus.

It was later found ${ }^{3}$ that the same technique could also be used for activating the cervical region, evoking muscle responses at a latency shorter than that evoked after cortical stimulation. By subtracting the latency of the cervical responses from that of the cortical ones, central motor conduction time can be measured.

We applied the technique of cortical and cervical stimulation in a group of subjects with motor deficits due to focal hemispheric infarction.

\section{Material and methods}

Twenty patients (mean age $64 \cdot 5 \pm 7 \cdot 4$, range $47-72$ years) with a unilateral upper motor neuron syndrome admitted to a rehabilitation unit for physiotherapy, and 20 normal subjects of comparable age and height (mean age $59 \cdot 2 \pm 9$,

Address for reprint requests: Dr Alfredo Berardelli, V Clinica Neurologica, Viale dell'Universita' 30, 00185 Roma, Italia.

Received 4 July 1986 and in revised form 6 November 1986. Accepted 13 November 1986 range 34-65 years) were studied. The patients had a hemispheric lesion of ischaemic origin and the study was carried out 1 to 22 months after the stroke, when the motor deficit was in the chronic stage. The clinical condition of the patients and the CT localisation of the lesion are summarised in table 1 . All the patients had abnormal plantar responses. All the subjects gave informed consent to the study, which was approved by the local ethical committee.

Stimulation of the motor cortex and cervical region was carried out with an electrical stimulator (Digitimer model 180) supplying a maximum of 750 volts. The stimulus time constant was either 50 or $100 \mu \mathrm{s}$. Cortical electrodes were placed on the scalp, the cathode on the vertex and the anode on the hand motor area of both sides $(7 \mathrm{~cm}$ down a line between the vertex and the auditory meatus). Normal subjects were only stimulated on one side. Cervical electrodes were placed over the spinous processes, the cathode on T1 and the anode on C4. Muscle action potentials (MAPs) were recorded bipolarly by surface electrodes on the biceps and thenar muscles, and stored on OTE Biopotential Analyzer Software Interactive System (BASIS).

In patients with hemiparesis stimulation of the damaged hemisphere was performed at rest and during a slight voluntary contralateral effort, in order to facilitate the MAPs. ${ }^{3}$ The facilitation only requires a minor degree of voluntary effort. The same degree of strength was exerted during stimulation of the undamaged hemisphere. Cervical stimulation was performed at rest since voluntary contraction does not affect the parameters of response. ${ }^{3}$ Eight MAPs responses were obtained from each stimulation site. Threshold, latency, duration and amplitude of the responses were measured. The MAPs were considered abnormal when they were absent at maximal stimulation or their latency exceeded the range of normal controls. Central conduction time (CCT) was obtained by subtracting the latency of MAPs evoked from the cortex (cortical MAPs) from the 


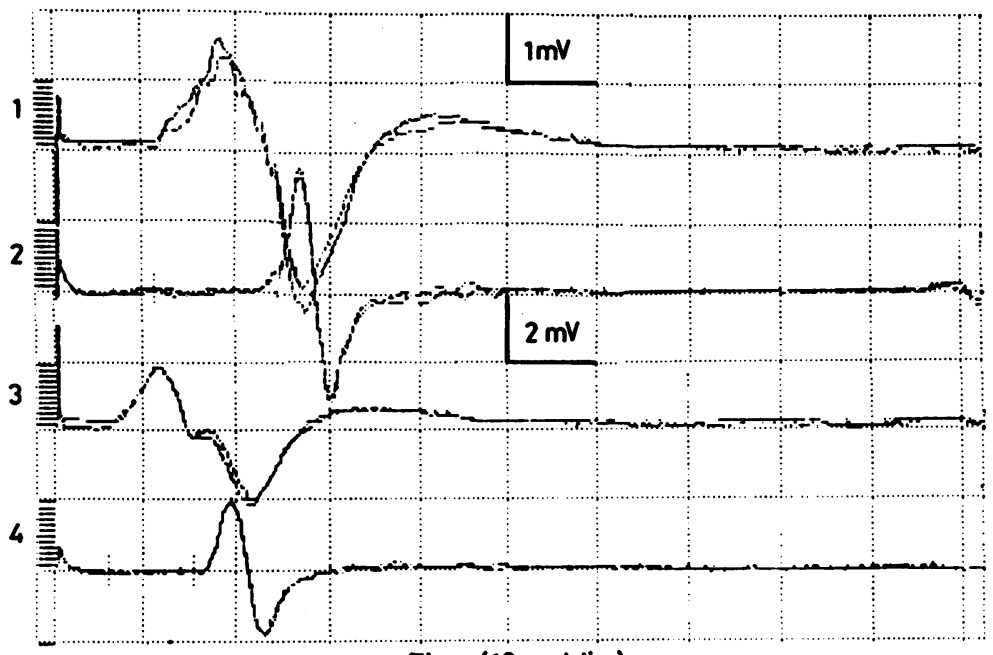

Time (10ms/div.)

Fig 1 Muscle action potential (MAPs) in a normal subject. Stimulation of the motor cortex ( 1 and 2) and cervical region ( 3 and 4). Responses from the biceps, first and third trace; and responses from thenar muscles, second and fourth trace. Superimposition of two single potentials.

latency of MAPs evoked from cervical region (cervical MAPs). Conduction velocity in the median nerve and amplitude of MAPs evoked by median nerve stimulation in the abductor pollicis brevis were measured bilaterally.

\section{Results}

In normal subjects, at rest and during voluntary effort motor cortex and cervical stimulation evoked MAPs in biceps and thenar muscles. The latencies of cortical and cervical MAPs are shown in table 2. The duration of MAPs on cortical stimulation was $19.0 \pm 3.0 \mathrm{~ms}$ in the contralateral biceps and $16.5 \pm 3.0 \mathrm{~ms}$ in contralateral thenar muscles (fig 1 (1) and (2)). The amplitude of the cortical MAPs varied widely accord ing to the degree of effort, and was not considered a reliable parameter. The same variability was observed

Table 1 Clinical features of patients

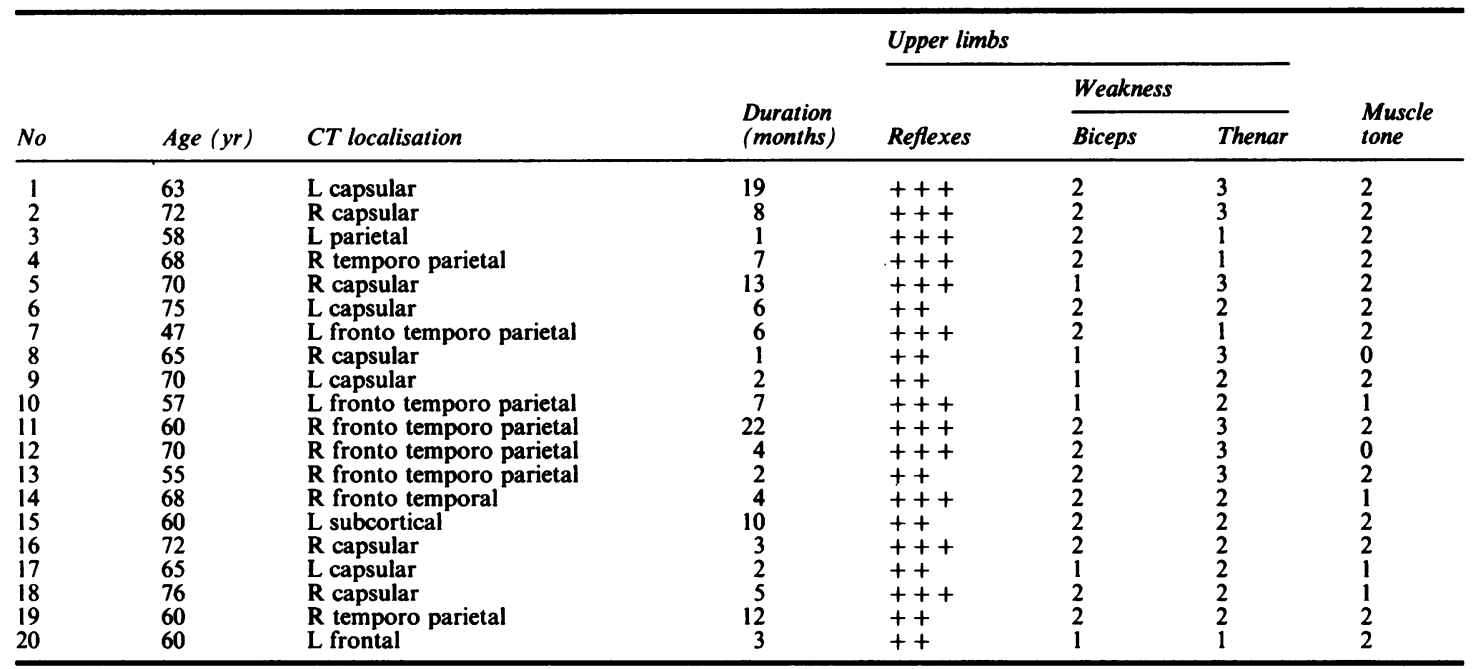

Tendon reflexes: + normal, ++ increased, +++ increased with clonus.

Weakness: 1 moderate, 2 severe, 3 paralysis.

Muscle tone: 0 reduced, 1 normal, 2 increased. 
for stimulation threshold. Cervical stimulation evoked on both sides MAPs of $20.2 \pm 2.5 \mathrm{~ms}$ in the biceps and $15.0 \pm 2.0 \mathrm{~ms}$ in the thenar muscles (fig 1 (3) and (4)). Median nerve stimulation elicited normal MAPs in the abductor pollicis brevis.

In patients, cortical MAPs from the undamaged hemisphere were normal except for patients nos 18 and 20, who showed a slight increase of CCT for thenar muscle (case no 18: $7.7 \mathrm{~ms}$; case no $20: 7.0 \mathrm{~ms}$ ). Cervical stimulation evoked MAPs of normal latency and duration in all the patients except nos 12 and 15 in whom the cervical MAPs were slightly delayed in the thenar muscles $(17.3 \mathrm{~ms}$ in patient $12 ; 17.7$ and $17.1 \mathrm{~ms}$ in patient 15 ). In these two patients the electromyographic studies revealed neurogenic signs in muscles innervated by seventh and eight cervical roots. In all the patients median nerve stimulation evoked MAPs of normal latency and duration.
Stimulation of the damaged hemisphere at maximal voltage did not elicit contralateral MAPs in 15 out of 20 patients (nos 1 to 15 ) (fig 2). In two patients (nos 16 and 17) the cortical MAPs were absent in the biceps and normal in the thenar muscle. In three patients the cortical MAPs were delayed in latency in both muscles (nos 18 and 19) or in the biceps (no 20); the central conduction time was prolonged (case no 18: $6.7 \mathrm{~ms}$ for biceps and $11.1 \mathrm{~ms}$ for thenar muscle; case no 19: $14.2 \mathrm{~ms}$ for biceps and $13.6 \mathrm{~ms}$ for thenar muscle; case no 20: $7.0 \mathrm{~ms}$ for biceps muscle).

The latencies of cortical and cervical MAPs and the CCT are listed in table 2.

Latency changes were generally accompanied by reduction in duration (case no 18: $10 \mathrm{~ms}$ for thenar muscle; case no 19: $12 \mathrm{~ms}$ for the biceps and $10 \mathrm{~ms}$ for thenar muscles; and case no $20: 11 \mathrm{~ms}$ for the biceps muscle).

Table 2 MAPs after cortical and spinal stimulation

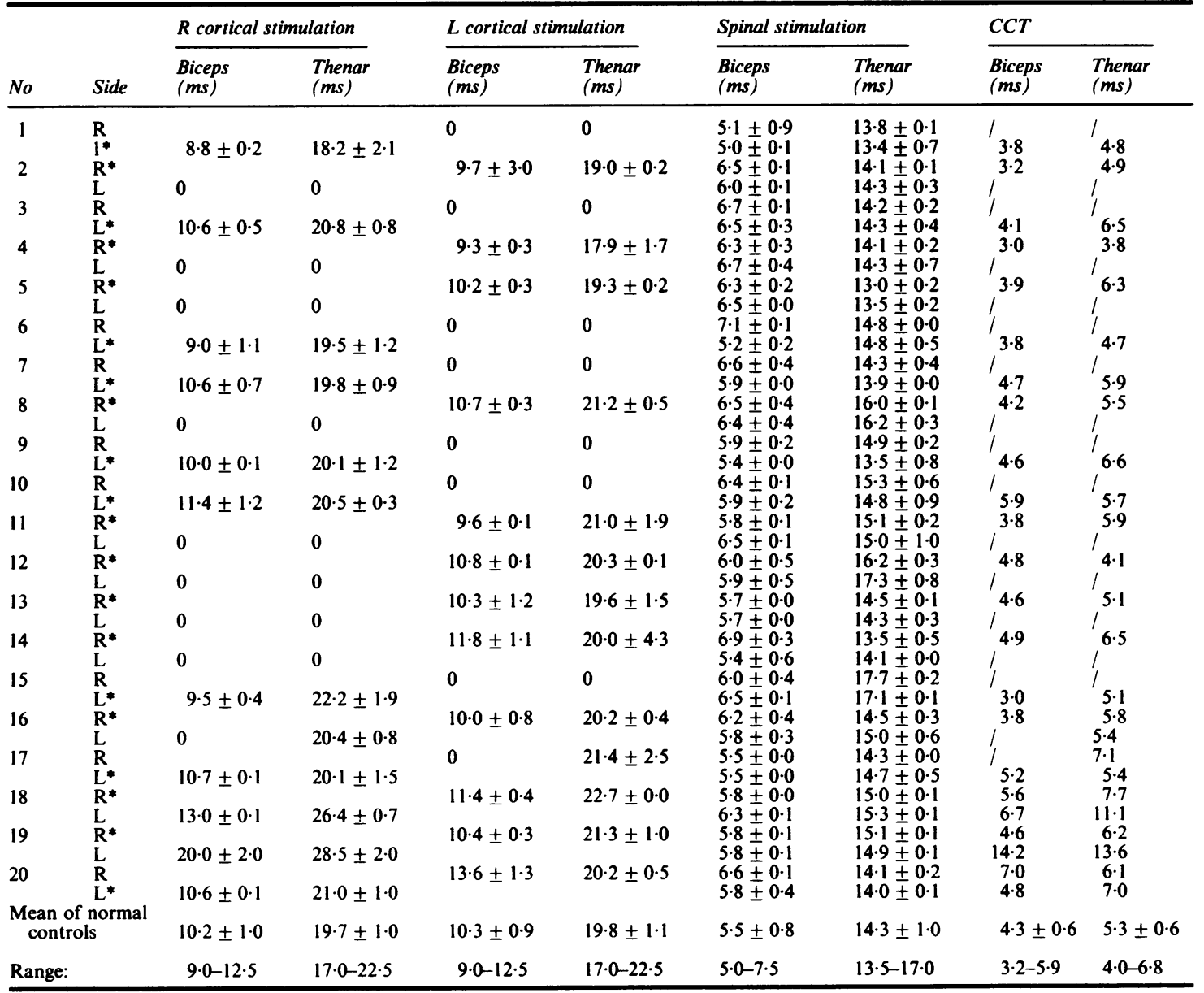

Values are mean \pm 1 SD.

* = site of hemispheral involvement. 


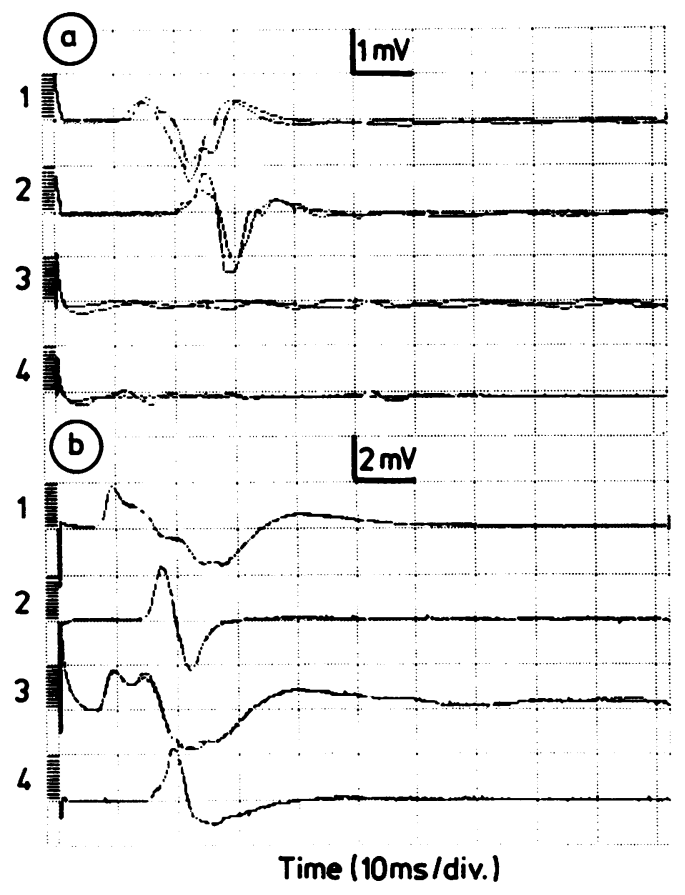

Fig 2 Muscle action potential (MAPs) in patient no 3. (A): Cortical stimulation. MAPs are normal in (1) right biceps and in (2) right thenar muscles after stimulation of the left cortex, but absent in (3) left biceps and (4) left thenar muscles after stimulation of the right cortex. $(B)$ : Cervical stimulation. MAPs are normal in (1) right biceps, in (2) right thenar, in (3) left biceps and in (4) left thenar. Superimposition of two single potentials.

\section{Discussion}

In the intact man, single pulse, high voltage stimulation of the motor cortex and of the spine ${ }^{23}$ activates the descending pathways and allows one to measure the central motor delay. Large efferent neurons of the motor cortex are probably excited since the latency is compatible with conduction through the fast corticospinal axons. ${ }^{4}$ The precise site of action of the stimulus has not been established. Cervical stimulation may excite the spinal motoneurons, the spinal interneurons or the ventral roots. The observation that the threshold and amplitude of the responses are not enhanced by voluntary contraction suggests that activation takes place at the level of the ventral roots. Mills and Murray ${ }^{5}$ have recently provided evidence that stimulation of the cervical region excites the motor roots at their exit from the spinal canal.

In 18 out of 20 patients studied the CCT was normal in the unaffected hemisphere. In two patients there was a slight increase of the CCT and this may be due to a lesion not demonstrated by clinical and radiological investigation.

The MAPs evoked by stimulation of the affected hemisphere and the CCT were constantly abnormal. The most common abnormality was absence of the potential at maximal voltage, a finding never observed in normal subjects or in the unaffected hemisphere of patients. Increased latency and decreased duration also seemed to be reliable indicators of corticospinal involvement, provided that the patients are able to produce a voluntary effort. The recordings from biceps were slightly more sensitive than those from thenar muscle since biceps MAPs were constantly abnormal while thenar MAPs were normal in three patients. Although response amplitude is to some extent related to response duration it varies considerably according to the degree of effort made by the subject, and is not in our opinion a reliable parameter. The same holds for the response threshold.

The cervical MAPs were normal in 18 and delayed in two patients. In these two patients there were EMG signs of a neurogenic lesion, a finding not uncommon in patients with hemiplegia and attributed to an associated entrapment or traction neuropathy or radiculopathy. ${ }^{6}$

It was difficult to correlate the degree of neurophysiological and clinical impairment since cortical MAPs were absent even in patients with incomplete motor deficit. For the same reason, no correlation was found with proximal and distal motor weakness. However, the five patients who still displayed cortical MAPs showed a less severe motor deficit.

Abnormal central motor conduction has been described in multiple sclerosis ${ }^{78}$ and motor neuron disease. ${ }^{9}$ In these conditions, the MAPs evoked from the motor cortex are usually delayed. This abnormality is attributed to demyelination with conduction block and to degeneration of the corticospinal fibres. After a stroke, the descending pathways are interrupted and this may explain why, in the majority of cases, the responses disappear. Increased latency and shortened duration of the residual cortical MAPs may be due to defective spatial summation of the corticospinal projection.

The finding that cortical responses could be absent even in patients in whom voluntary movement was still possible may suggest that movement occurred through non pyramidal pathways or through the small pyramical tract fibres.

\section{References}

1 Gualtierotti T, Paterson AS. Electrical stimulation of the unexposed cortex. J Physiol (Lond) 1954;125:278-91.

2 Merton PA, Morton HB. Stimulation of the cerebral 
cortex in the intact human subjects. Nature 1980; 285:227.

3 Merton PA, Morton HB, Hill DK, Marsden CD. Scope of a technique for electrical stimulation of human brain, spinal cord, and muscle. Lancet 1982;2:597-600.

4 Phillips CG, Porter R. Corticospinal Neurons. London: Academic Press, 1977:135-8.

5 Mills KR, Murray NMF. Electrical stimulation over the human vertebral column: which neural elements are excited? Electroencephalogr Clin Neurophysiol 1986; 63:582-9.

6 Chokroverty S, Medina J. Electrophysiological study of hemiplegia. Arch Neurol 1978;35:360-3.

7 Cowan JMA, Rothwell JC, Dick JPR, Day BL, Thompson PD, Marsden CD. Abnormalities in the central motor pathways in multiple sclerosis. Lancet 1984;ii:304-7.

8 Mills KR, Murray NMF. Corticospinal tract conduction time in multiple sclerosis. Ann Neurol 1985;18:601-5.

9 Berardelli A, Inghilleri M, Formisano R, Accornero N, Manfredi M. Central and peripheral motor delays in motor neuron disease. J Neurol Neurosurg Psychiatry (in press). 